Article

\title{
Rv0613c/MSMEG_1285 Interacts with HBHA and Mediates Its Proper Cell-Surface Exposure in Mycobacteria
}

\author{
Romain Veyron-Churlet*(D), Vincent Dupres, Jean-Michel Saliou, Frank Lafont, \\ Dominique Raze ${ }^{(D)}$ and Camille Locht*
}

Université de Lille, CNRS UMR8204, INSERM U1019, Centre d'Infection et d'Immunité de Lille, Institut Pasteur de Lille, 59000 Lille, France; vincent.dupres@ibl.cnrs.fr (V.D.); jean-michel.saliou@pasteur-lille.fr (J.-M.S.); frank.lafont@ibl.cnrs.fr (F.L.); dominique.raze@ibl.cnrs.fr (D.R.)

* Correspondence: romain_veyron@yahoo.fr (R.V.-C.); camille.locht@pasteur-lille.fr (C.L.); Tel.: +33-32-087-1152 (R.V.-C.); +33-32-087-1151 (C.L.)

Received: 20 April 2018; Accepted: 29 May 2018; Published: 5 June 2018

\begin{abstract}
Heparin-binding haemagglutinin (HBHA) is a surface-exposed virulence factor of Mycobacterium tuberculosis and is involved in the binding of mycobacteria to non-phagocytic cells, allowing for extra-pulmonary dissemination of the bacilli. Despite its surface exposure, HBHA is not produced as a pre-protein containing a typical cleavable $\mathrm{N}$-terminal signal peptide and is thus likely secreted by a Sec-independent, as of yet unknown mechanism. Here, we used the bacterial adenylate cyclase two-hybrid system to identify the proteins encoded by rv0613c and mmpL14 as being able to interact with HBHA. Our study was focused on Rv0613c, as it showed more consistent interactions with HBHA than MmpL14. Deletion of its orthologous gene MSMEG_1285 in recombinant Mycobacterium smegmatis producing HBHA from $M$. tuberculosis resulted in the loss of proper surface exposure of HBHA, as evidenced by atomic force microscopy. Furthermore, the lack of MSMEG_1285 also abolished the clumping phenotype and rough colony morphology of the recombinant $M$. smegmatis and reduced its adherence to A549 epithelial cells. These phenotypes have previously been associated with surface-exposed HBHA. Thus, MSMEG_1285 is directly involved in the proper cell-surface exposure of HBHA. These observations identify MSMEG_1285/Rv0613c as the first accessory protein involved in the cell surface exposure of HBHA.
\end{abstract}

Keywords: tuberculosis; Mycobacterium; host-pathogen interaction; adhesin; cell wall

\section{Introduction}

Tuberculosis (TB) remains the leading global cause of infection-related mortality and morbidity, with 1.7 million deaths and an estimated incidence of 10.4 million cases in 2016 [1]. TB pathology is linked to the tight interplay between the human immune system and the persistence of the bacilli within the host. Mycobacterium tuberculosis $(M t b)$, the causative agent of human TB, produces virulence factors that contribute to its successful colonization of the host cells. Among them, the Heparin-binding haemagglutinin (HBHA) is a surface-exposed mycobacterial adhesin [2,3] that is able to bind heparan sulfate [4]. It is not involved in the interaction with macrophages but is necessary for the mycobacterial interactions with type II alveolar epithelial cells in vitro [5], and heparan sulfate moieties on proteins, such as Syndecan-4, are implicated in mycobacterial internalization by lung epithelial cells [6]. Although HBHA is dispensable for initial pulmonary colonization, the protein is required for extrapulmonary dissemination of the bacilli in mice [5]. It is a highly methylated protein in its C-terminal part [7] and its complex methylation profile plays a direct role in the immunogenicity and 
recognition of HBHA by T cells from $M t b$-infected subjects [8]. An orthologous protein is also present in fast-growing and non-pathogenic Mycobacterium smegmatis (Msmeg). However, HBHA_Msmeg is not involved in epithelial cell adhesion and does not display high affinity for heparin [9]. Thus, HBHA most likely underwent a functional divergence during evolution $[9,10]$. Despite its cell surface exposure in $M t b$ [2], HBHA lacks a canonical signal peptide, usually required for protein transport through bacterial plasma membranes. Thus, HBHA crosses the mycobacterial membrane by an unknown mechanism that is independent of the classical signal sequences peptides.

In this study, as an approach to unravel HBHA cell surface exposure, we identified the proteins encoded by ro0613c and mmpL14, as the first proteins able to interact with HBHA. Recombinant Msmeg $\mathrm{mc}^{2} 155$ expressing $h b h A \_M t b$ was found to auto-aggregate, to grow as rough colonies and to infect epithelial cells, whereas the deletion of MSMEG_1285, the orthologous gene of rv0613c in Msmeg mc $^{2} 155$, abolished these phenotypes. Furthermore, HBHA_Mtb was detected by atomic force microscopy at the surface of recombinant Msmeg $\mathrm{mc}^{2} 155$ but not of the recombinant $\triangle M S M E G \_1285$ mutant, indicating an important role for Rv0613c/MSMEG_1285 in the cell-surface exposure of HBHA.

\section{Results}

\subsection{The N-Terminal Part of HBHA Interacts with Rv0613c and MmpL14}

To identify protein partners able to interact with HBHA, we used the Bacterial Adenylate Cyclase Two-Hybrid (BACTH) system with pKT25_hbhA as a bait to screen a Mtb Erdman library contained in pUT18C [11]. The initial bacterial colonies selected based on their red color on MacConkey agar were further transferred onto fresh selective plates to confirm the phenotype. DNA was then extracted from the selected colonies and the pUT18C inserts were sequenced. This led to the identification of a portion of the rv0613c and mmpL14 genes. These portions code for amino acids 314 to 550 and 519 to 749 of Rv0613c and MmpL14, respectively. The rv0613c gene from Mtb Erdman is 100\% identical to that of $M t b$ H37Rv. The $m m p L 14$ gene is absent from Mtb H37Rv but completely present in Mtb Erdman [12,13]. pUT18C containing the truncated rv0613 $c_{314-550}$ or mmpL14519-749 were re-introduced into Escherichia coli DHM1 containing pKT25_hbhA to confirm the phenotype in the BACTH system. For the bacteria expressing rv0613c $c_{314-550}$, all the colonies displayed a red color on MacConkey agar plates, whereas for the bacteria expressing mmpL14 ${ }_{519-749}$, the red labeling was more heterogeneous (Figure 1A). As expected, E. coli DHM1 containing pKT25_hbhA together with empty pUT18C did not yield any red colonies, nor did E. coli DHM1 containing empty pKT25 together with pUT18C_rv0613c314-550 or pUT18C_mmpL14519-749 (Figure 1A).

The C-terminal part of HBHA contains the heparin-binding domain (HBD) [14], a 39 amino acid region enriched in lysine, alanine and proline residues, which could potentially lead to non-specific interactions with other proteins. In order to determine whether this C-terminal domain of HBHA is responsible for its interactions with Rv0613 and/or MmpL14, we introduced the partial hbhA DNA fragments coding for HBHA $_{1-109}$, devoid of its linker and HBD regions into pKT25 [15]. When this construct was used as a bait in the BACTH system, co-expressed with either ro0613c $314-550$ or mmpL14519-749, it yielded red colonies in both cases, again more consistently with ro0613c314-550 than with mmpL14 ${ }_{519-749}$ (Figure 1B). These results indicate that the N-terminal moiety of HBHA interacts with Rv0613 and MmpL14. In addition, as only the C-terminal part of HBHA is methylated, this implies that methylation does not seem to be important for HBHA interactions with Rv0613c or MmpL14. All these interactions were further confirmed by using LB agar plates supplemented with IPTG and X-gal (Supplementary Figure S1). 
A

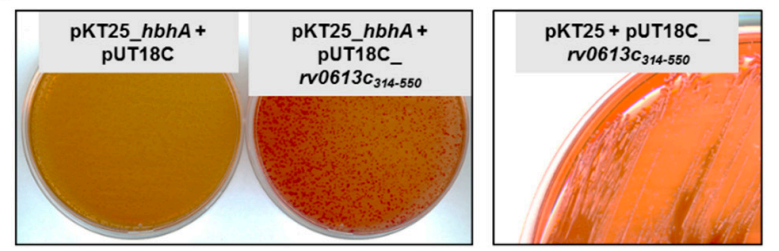

B

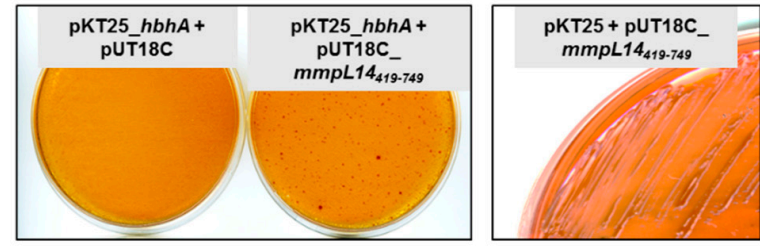

C

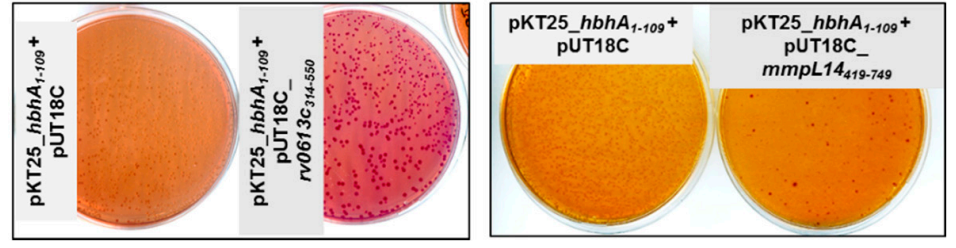

Figure 1. HBHA interacts with Rv0613c and MmpL14 in the BACTH system. (A) Red colony phenotype observed on MacConkey agar plates for E. coli transformed with pKT25_hbhA and pUT18C_rv0613c314-550 (middle plate) in contrast to the E. coli transformed with pKT25_hbhA and pUT18C (left plate) or pKT25 and pUT18C_rv0613c $314-550$ (right plate); (B) Red colony phenotype observed on MacConkey agar plates for E. coli transformed with pKT25_hbhA and pUT18C_mmpL14519-749 (middle plate) in contrast to the E. coli transformed with pKT25_hbhA and pUT18C (left plate) or pKT25 and pUT18C_mmpL14519-749 (right plate); (C) Red colony phenotype observed on MacConkey agar plates for E. coli transformed with pKT25_hbhA $A_{1-109}$ and pUT18C_rv0613c314-550 or pUT18C_mmpL14519-749 (right plates) in contrast to the E. coli transformed with pKT25_hbh $A_{1-109}$ and pUT18C (left plates).

\subsection{Rv0613c Is Conserved within the Mtb Complex and Contains a SEC-C Motif}

The rv0613c gene codes for a protein of 855 amino acids. Like HBHA, Rv0613c has orthologues only in other Actinobacteria and is strictly conserved among the Mtb complex (Figure 2A). Among fast-growing mycobacteria, the Msmeg protein MSMEG_1285 is the closest orthologues of Rv0613c, displaying 59\% sequence identity with full-length Rv0613c and 66\% sequence identity with Rv0613c $314-550$ (Figure 2B). No orthologue was found in M. leprae, M. marinum, M. ulcerans, M. avium, M. fortuitum and M. chelonae.

In silico analyses of the Rv0613c sequence revealed poor folding predictions [16]. However, $\mathrm{Rv} 0613 \mathrm{c}$ is annotated as a protein containing a SEC-C motif (around 20 amino acids), which is notably found in bacterial preprotein translocases. MSMEG_1285 also contains a SEC-C motif and an additional TetratricoPeptide Repeat (TPR) domain that may be involved in protein-protein interaction (Figure 2B). TPR-containing proteins are associated with various virulence mechanisms in several bacterial pathogens [17]. A homologous region of the TPR domain is present in almost its entire length 
in Rv0613 $c_{314-550}$ (Figure 2B). Therefore, this region may potentially be involved in the HBHA-Rv0613c interaction, although Rv0613c was not annotated as a protein containing such a domain, probably due to some sequence divergence between $M t b \mathrm{H} 37 \mathrm{Rv}$ and $M s m e g \mathrm{mc}^{2} 155$ in the N-terminal part of the TPR domain.

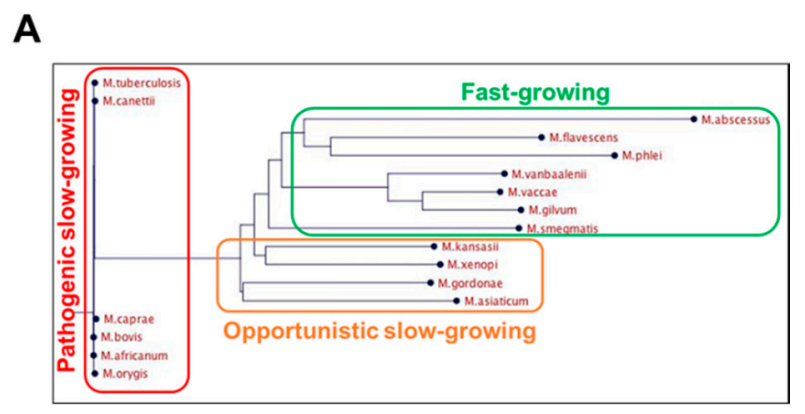

B

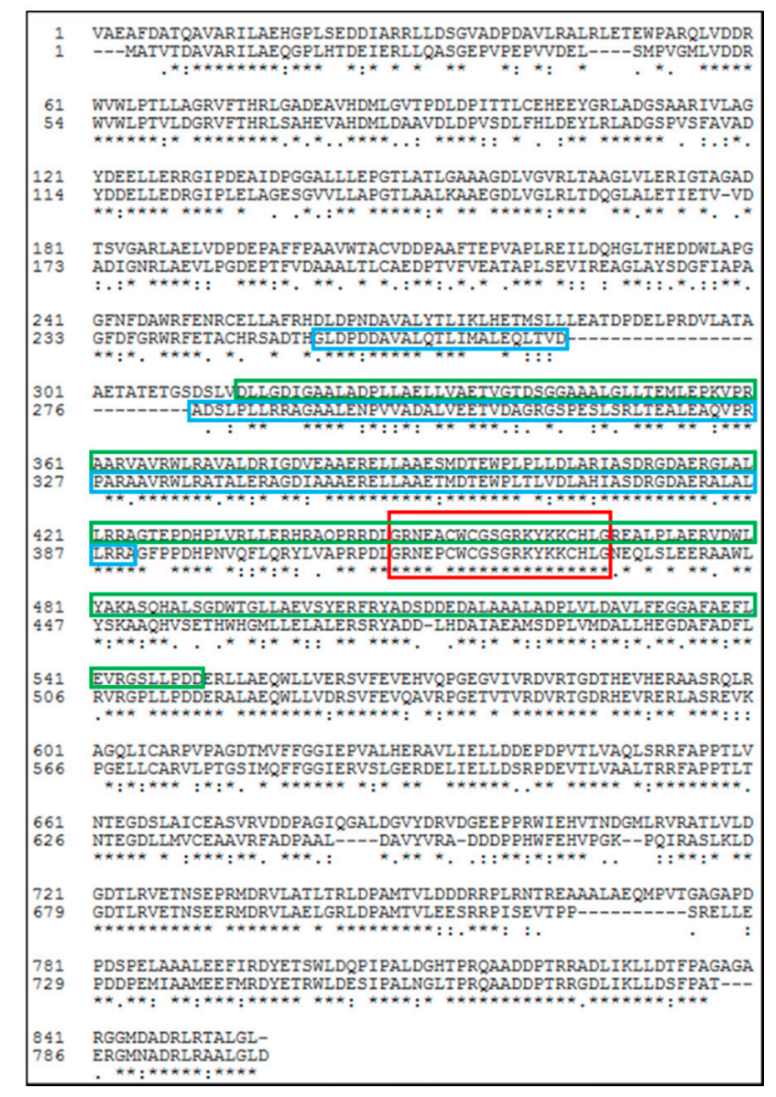

Figure 2. MSMEG_1285 is an orthologue of Rv0613c. (A) Phylogenetic tree representing Rv0613c homologues among different mycobacteria; (B) Sequence alignment of Rv0613c (first line) and MSMEG_1285 (second line) using Clustal Omega. The red rectangle corresponds to the SEC-C motif. The region interacting with HBHA of Rv0613 $314-550$ is shown in the green rectangles, and the TPR of MSMEG_1285 is shown in the blue rectangles.

\subsection{Generation of a $\triangle M S M E G \_1285$ Mutant in Msmeg $m c^{2} 155$}

To characterize the role of Rv0613c/MSMEG_1285, we used fast-growing Msmeg as a model system. A MSMEG_1285 deletion mutant (DMSMEG_1285) was generated in Msmeg mc $^{2} 155$, as well as its complemented derivative (Supplementary Figure S2A,B), proving that MSMEG_1285 is dispensable for Msmeg $\mathrm{mc}^{2} 155$ growth in vitro. The absence of $M S M E G \_1285$ and the complementation were 
verified using two different sera from Rv0613c-immunized mice (Supplementary Figure S2C). Moreover, the total amount of intracellular HBHA_Msmeg was not impacted by the deletion of MSMEG_1285 (Supplementary Figure S2D).

\subsection{MSMEG_1285 Is Necessary for the Proper Cell-Surface Exposure of HBHA_Mtb}

Atomic Force Microscopy (AFM) has been successfully used to examine the cell-surface exposure of HBHA in Mycobacterium bovis bacillus Calmette-Guérin (BCG) and its interaction with heparin coated on the AFM tip [18]. HBHA is a highly abundant protein, with up to $1 \%$ of total soluble proteins of BCG Pasteur. In contrast, HBHA_Msmeg is less abundant, explaining why the presence of an orthologous protein in Msmeg remained elusive for years [9]. In addition, the affinity between HBHA_Msmeg and heparin is much lower than the affinity between HBHA_Mtb and heparin [9]. Therefore, to monitor HBHA interaction with heparin, we transformed Msmeg $\mathrm{mc}^{2} 155$ and the $\triangle M S M E G_{-} 1285$ with pMV361_hbhA_EGFP, allowing heterologous expression of $h b h A \_M t b$. The fusion with EGFP did not affect cell surface-exposure of HBHA in BCG Pasteur [19] and was further used to distinguish between endogenous HBHA_Msmeg and the recombinant HBHA_Mtb.

When grown in 7H9 plus OADC without detergent, the absence of MSMEG_1285 was found to significantly decrease the binding between heparin and HBHA_M $t b$ produced in $M s m e g$, as evidenced by the poor interaction of HBHA_Mtb produced in the $\triangle M S M E G \_1285$ mutant with the AFM heparin-coated tip. Only $47 \%$ of the measurements did not detect adhesion events in recombinant Msmeg $\mathrm{mc}^{2} 155$ producing HBHA_Mtb (Figure 3B), whereas this increased to $91 \%$ of measurements in the $\triangle M S M E G \_1285$ mutant (Figure 3D). Without recombinant production of HBHA_Mtb, the wild-type strain or the $\triangle M S M E G \_1285$ mutant barely interacted with the AFM heparin-coated tip as $97 \%$ of the measurements did not detect adhesion events in either case (Figure 3A,C), confirming that HBHA_Msmeg has low affinity for heparin and therefore does not interfere with AFM measurement. When grown in Sauton medium (supplemented with soft detergent $0.025 \%$ tyloxapol), the difference between the two recombinant strains producing $h b h A \_M t b$ was confirmed, with $24 \%$ and $79 \%$ of measurements not detecting any adhesion events for Msmeg mc $^{2} 155$ and the $\triangle M S M E G \_1285$ mutant, respectively (Supplementary Figure S3B,D). Here again, recombinant production of HBHA_Mtb was necessary, as $97 \%$ of the measurements with the wild-type strain did not show any adhesion events (Supplementary Figure S3A) while 81\% of the measurements with the $\triangle M S M E G \_1285$ mutant did not give any adhesion events (Supplementary Figure S3C). These results thus demonstrate that MSMEG_1285 is necessary for the proper cell-surface exposure of HBHA_Mtb in Msmeg $\mathrm{mc}^{2} 155$.

\subsection{Loss of Auto-Aggregation in $\triangle M S M E G \_1285$ Expressing $h b h A \_M t b$}

Surface-exposed HBHA_Mtb has been shown to cause auto-aggregation of mycobacteria [2]. To assess the role of MSMEG_1285 in auto-aggregation, recombinant $M s m e g \mathrm{mc}^{2} 155$ and $\triangle M S M E G \_1285$ producing HBHA_Mtb were grown in 7H9 medium supplemented with OADC in the absence of detergent. Production of recombinant HBHA_Mtb in Msmeg $\mathrm{mc}^{2} 155$ induced clumping of the mycobacteria, consistent with previous work [2] (Figure 4A). In contrast, in the same culture conditions, the $\triangle M S M E G \_1285$ mutant producing HBHA_Mtb failed to form clumps (Figure 4A). This phenotype was related to the recombinant production of HBHA_Mtb as the non-recombinant strains did not display any difference in liquid cultures (Supplementary Figure S4A). When grown on 7H11 agar plates, the colonies of the $\triangle M S M E G \_1285$ mutant expressing $h b h A \_M t b$ displayed a smooth and round phenotype, whereas $M s m e g \mathrm{mc}^{2} 155$ expressing $h b h A \_M t b$ displayed a rough phenotype (Figure 4B). Again, this phenotype was related to the recombinant production of HBHA_Mtb as the non-recombinant strains grew as rough colonies on 7H11 agar plates (Supplementary Figure S4B). As the level of recombinant HBHA_Mtb in fusion with EGFP (48.4 kDa for hybrid protein) or endogenous HBHA_Msmeg $(24.6 \mathrm{kDa})$ production by $M s m e g$ was not impacted by the $\triangle M S M E G \_1285$ deletion (Figure $4 \mathrm{C}$ ), this infers that the clumping and the rough phenotypes are mediated by a MSMEG_1285-dependent surface exposure of HBHA_Mtb. 
A
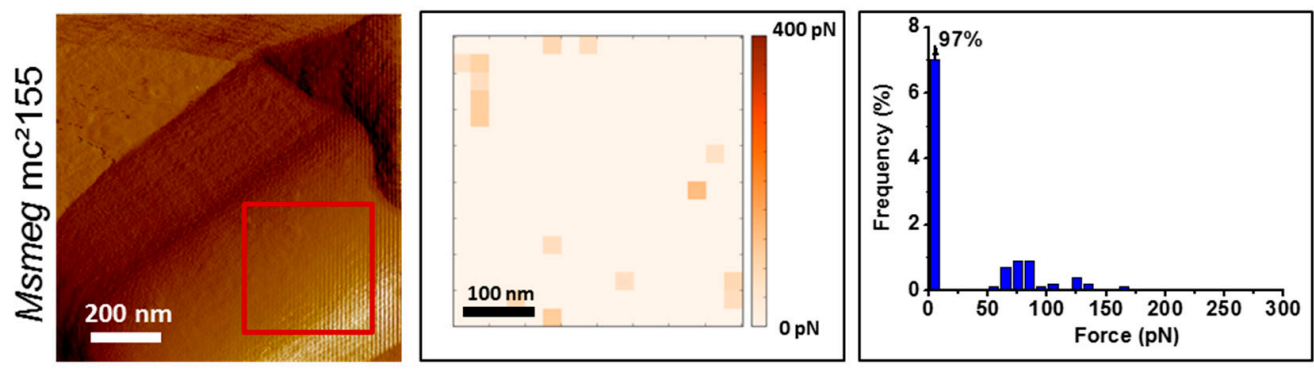

B
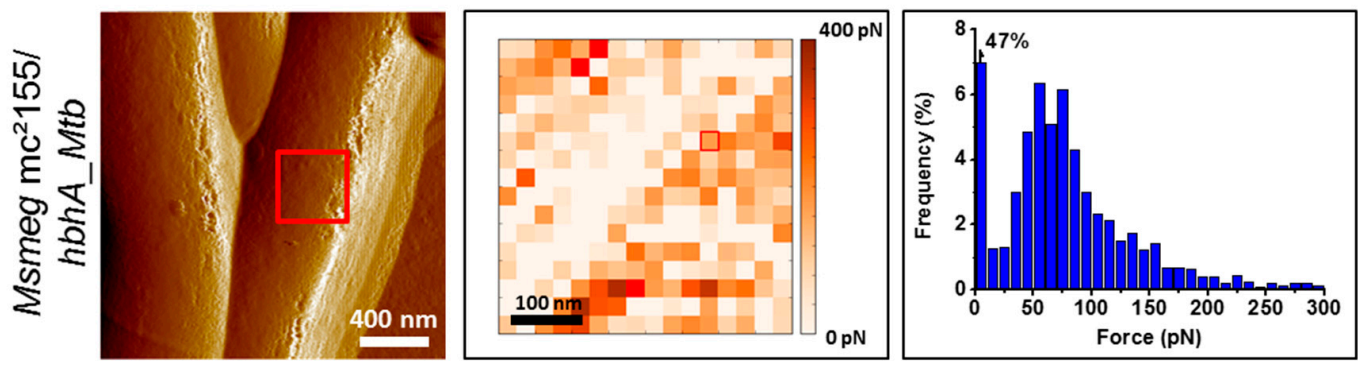

C
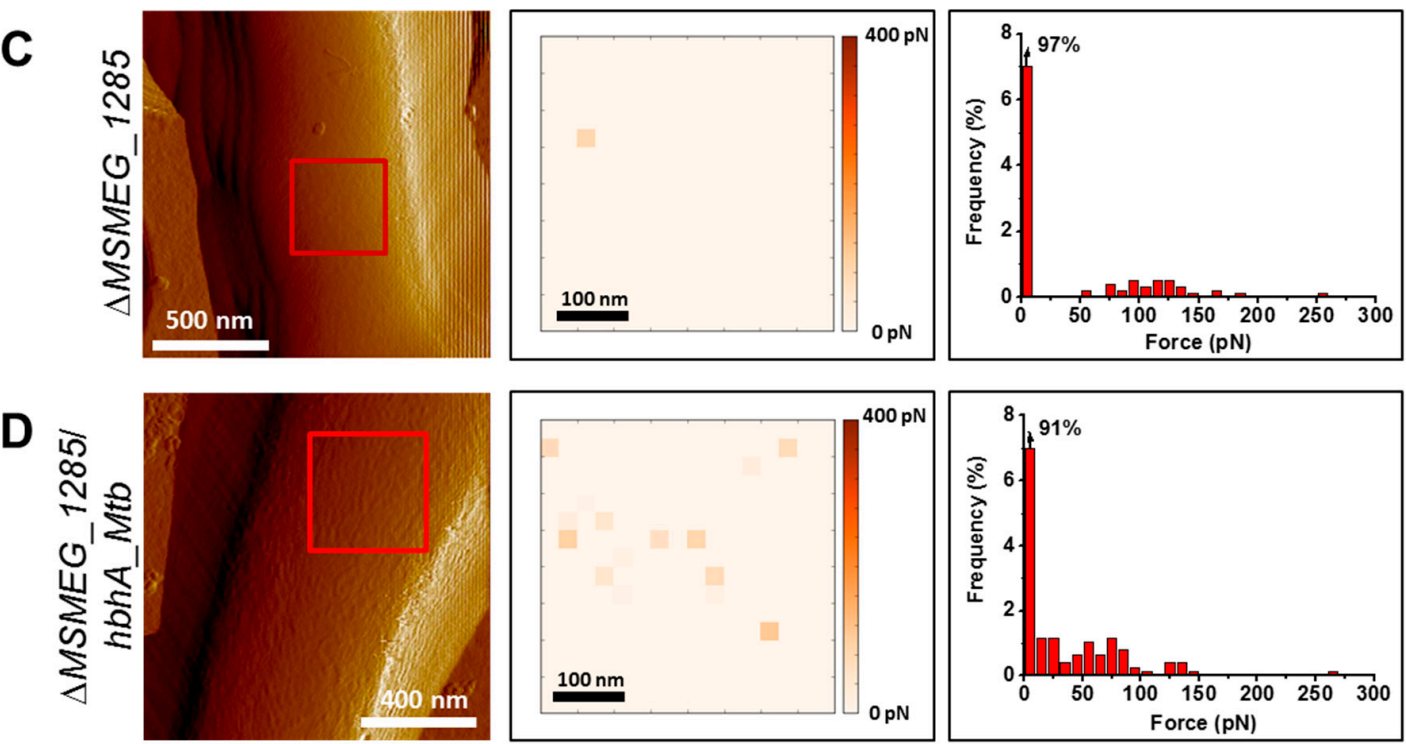

Figure 3. Deletion of MSMEG_1285 in Msmeg $\mathrm{mc}^{2} 155$ impacts the cell-surface exposure of HBHA_Mtb. AFM tomographic image (left panel), representative spatially-resolved map of adhesion forces recorded with AFM heparin-coated tip (medium panel) and corresponding histogram (right panel, obtained from 756 force curves) for (A) the wild-type strain of $M s m e g$, (B) Msmeg mc ${ }^{2} 155$ expressing $h b h A \_M t b$, (C) the $\triangle M S M E G \_1285$ mutant and (D) the $\triangle M S M E G \_1285$ mutant expressing $h b h A \_M t b$. All the strains were cultured in $7 \mathrm{H} 9$ supplemented with OADC and without detergent. For each image, the red square corresponds to the area scanned for the determination of one adhesion map. 
A

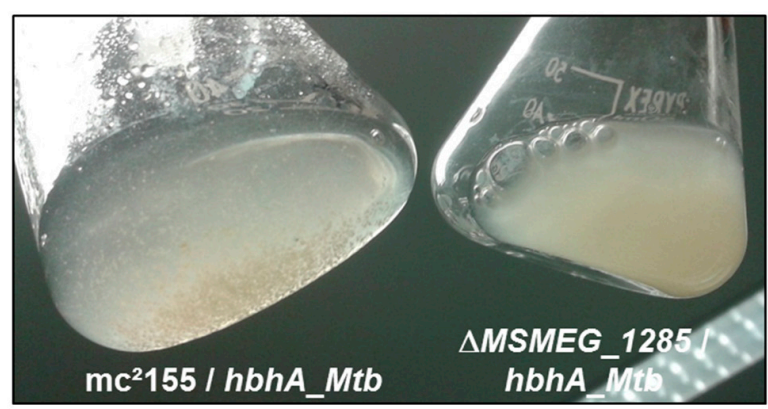

B

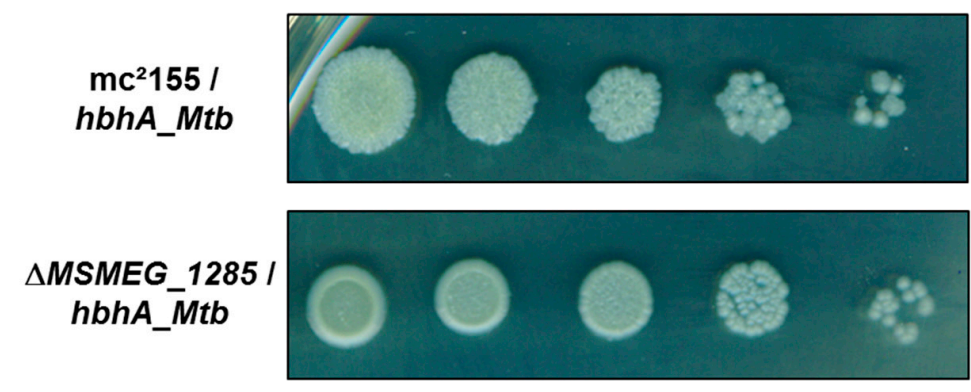

C

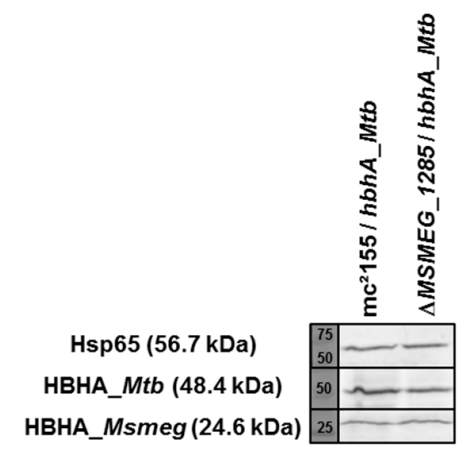

Figure 4. Heterologous expression of $h b h A \_M t b$ in the $\triangle M S M E G \_1285$ mutant affects auto-aggregation and colony morphology. (A) Overnight cultures in 7H9 supplemented with OADC and without detergent of Msmeg $\mathrm{mc}^{2} 155$ and the $\triangle M S M E G \_1285$ mutant, both expressing $h b h A \_M t b$; (B) Serial dilution of Msmeg $\mathrm{mc}^{2} 155$ (upper series) and the $\triangle M S M E G \_1285$ mutant (lower series), both expressing $h b h A \_M t b$, on 7H11 agar plates; (C) Western-blot analysis using monoclonal antibodies anti-Hsp65 (upper panel), anti-HBHA VF2 (medium panel) and anti-HBHA D2 (lower panel) on $40 \mu \mathrm{g}$ of total lysates from Msmeg $\mathrm{mc}^{2} 155$ and the $\triangle M S M E G \_1285$ mutant, both expressing $h b h A \_M t b$.

\subsection{MSMEG_1285 Is Necessary for Full Infectivity of Msmeg $m c^{2} 155$ Expressing $h b h A \_M t b$}

It has previously been shown that HBHA_M $t b$ mediates the interaction between $M t b$ and epithelial cells, leading to extrapulmonary dissemination of the bacilli [5]. Using the epithelial cell line A549, we examined the impact of $M S M E G \_1285$ deletion on HBHA_Mtb-mediated cytoadherence. Therefore, Msmeg $\mathrm{mc}^{2} 155, \triangle M S M E G \_1285$ and their recombinant HBHA_Mtb-producing derivatives were used to infect A549 cells. A difference was observed after $4 \mathrm{~h}$ of infection (day 0), as a higher number 
of colony-forming units (CFU) were detected on cells incubated with recombinant $M s m e g \mathrm{mc}^{2} 155$ expressing $h b h A \_M t b$ compared to the recombinant $\triangle M S M E G \_1285$ mutant (Figure 5A). This difference was maintained after three days after of incubation (Figure 5A). This phenotype was dependent on HBHA_Mtb as the non-recombinant $\triangle M S M E G \_1285$ mutant did not differ from non-recombinant Msmeg $\mathrm{mc}^{2} 155$ or the complemented strain in its ability to infect A549 infectivity from day 0 to day 3 (Figure 5B), indicating that MSMEG_1285 is required for HBHA_Mtb-mediated adherence to epithelial cells.

A

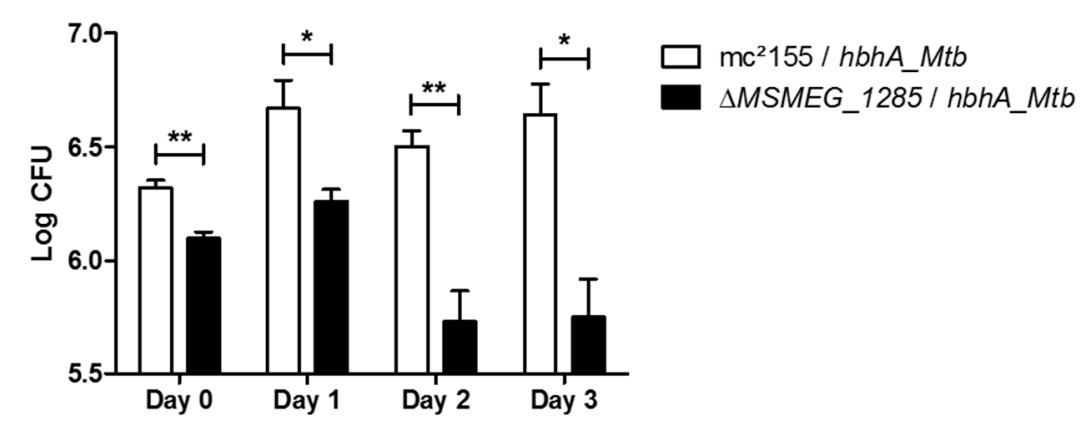

B

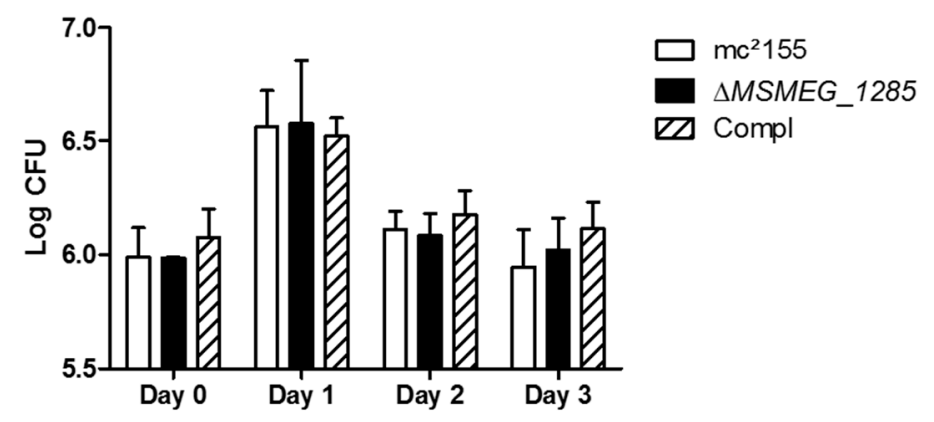

Figure 5. Heterologous expression of $h b h A \_M t b$ in the $\triangle M S M E G \_1285$ mutant impairs its infectivity towards A549 cells (A) Mean values of CFU counting for the recombinant M. smegmatis mc $^{2} 155$ (white bars) and $\triangle M S M E G \_1285$ mutant (black bars), both expressing $h b h A \_M t b$, after infection of A549 cells; (B) CFU counting for the non-recombinant M. smegmatis mc $^{2} 155$ (white bars), $\triangle M S M E G \_1285$ mutant (black bars) and complemented strain (striped bars), after infection of A549 cells. The two graphs are representative of two independent experiments. ${ }^{*}, p<0.05 ;{ }^{* *}, p<0.01$.

\subsection{Proteomic Analysis of the $\triangle M S M E G \_1285$ Mutant}

In order to determine whether any protein is affected in its localization by the deletion of MSMEG_1285, the protein content of the membrane (MB) and cell wall (CW) fractions of Msmeg $\mathrm{mc}^{2} 155$, the MSMEG_1285 mutant and the complemented strains, as well as the recombinant Msmeg strains expressing $h b h A \_M t b$, was examined by mass spectrometry analyses. Using a spectral counting approach with a ratio $\leq 0.2$ for the total number of spectra (mutant/wild type and mutant/complemented strain), no protein was lacking in the MB fraction of the $\triangle M S M E G \_1285$ mutant when compared with either the wild type or the complemented strain. However, surprisingly, using a spectral counting approach with a ratio $\geq 5$, one protein, MSMEG_3496, appeared to be 
enriched in the MB fraction of the $\triangle M S M E G_{-} 1285$ mutant in comparison with both the wild type and complemented strains (Table 1). Fourteen spectra for MSMEG_3496 were detected in the mutant, whereas only one spectrum was detected for recombinant Msmeg $\mathrm{mc}^{2} 155$ (Table 1). Thus, the absence of MSMEG_1285 led to an enrichment of MSMEG_3496 in the Msmeg MB fraction. However, this tendency was not confirmed in the CW fraction of recombinant strains (Table 1).

The total number of spectra for HBHA_Mtb was similar between recombinant Msmeg $\mathrm{mc}^{2} 155$ and $\triangle M S M E G \_1285$ mutant (Table 1), confirming that the phenotypes observed above were not due to differences in protein content of the $\mathrm{MB}$ or $\mathrm{CW}$ fractions, but were due to differences in HBHA cell-surface exposure between Msmeg $\mathrm{mc}^{2} 155$ and the MSMEG_1285-deficient mutant. As expected, MSMEG_1285 was not detected in the $\triangle M S M E G \_1285$ mutant.

Table 1. Total number of spectra (and unique peptides) for each protein in Membrane (MB) and Cell Wall (CW) fractions.

\begin{tabular}{|c|c|c|c|c|c|c|c|}
\hline Protein & MW & Fraction & $\mathrm{mc}^{2} 155$ & $\Delta M S M E G \_1285$ & Complemented & $\mathrm{mc}^{2} 155 / h b h A \_M t b$ & $\Delta M S M E G \_1285 / h b h A \_M t b$ \\
\hline \multirow{2}{*}{ MSMEG_3496 } & \multirow{2}{*}{106210} & MB & 1 (1) & $5(4)$ & 1 (1) & $1(1)$ & $14(12)$ \\
\hline & & $\mathrm{CW}$ & $4(3)$ & $9(6)$ & $4(3)$ & $48(22)$ & $15(10)$ \\
\hline \multirow{2}{*}{ MSMEG_1285 } & \multirow{2}{*}{88003} & MB & $1(1)$ & 0 & $4(3)$ & $3(3)$ & 0 \\
\hline & & $\mathrm{CW}$ & $1(1)$ & 0 & $11(8)$ & $2(2)$ & 0 \\
\hline \multirow{2}{*}{$\begin{array}{l}\text { MSMEG_0919 } \\
\text { (HBHA_Msmeg) }\end{array}$} & \multirow{2}{*}{24562} & MB & $28(12)$ & $37(13)$ & $27(11)$ & $13(7)$ & $23(11)$ \\
\hline & & $\mathrm{CW}$ & $15(9)$ & $16(10)$ & $16(7)$ & $7(5)$ & $13(8)$ \\
\hline \multirow{2}{*}{$\begin{array}{r}\text { Rv0475_EGFP } \\
\text { (HBHA_Mtb) }\end{array}$} & \multirow{2}{*}{48472} & MB & 0 & 0 & 0 & $17(14)$ & $14(10)$ \\
\hline & & CW & 0 & 0 & 0 & $4(4)$ & $5(4)$ \\
\hline
\end{tabular}

\section{Discussion}

Considering the importance of HBHA in $M t b$ pathogenesis [5], unraveling its biosynthesis and its function is a crucial step in understanding the ability of the bacilli to interact with epithelial cells and to undergo extrapulmonary dissemination. HBHA was previously detected at the cell-surface of the bacilli [2], although it lacks a canonical signal peptide for transport. Here, we describe Rv0613c and MmpL14 as the first proteins identified to interact with HBHA. Using the BACTH system, the HBHA interaction with Rv0613c was consistently strong, whereas the interaction was less consistent with MmpL14, as the red phenotype displayed by E. coli cells on McConkey agar plates in the BACTH system appeared to be more heterogeneous, in comparison with the HBHA-Rv0613 $c_{314-550}$ interaction. However, this heterogeneity could be the consequence of using a truncated version of MmpL14 (i.e., MmpL14 ${ }_{519-749}$ ) in BACTH system, and other portions of MmpL14 may be needed to retain full interaction with HBHA. Therefore, we focused this study on Rv0613c, for which we could identify its homolog in Msmeg as MSMEG_1285.

HBHA has several functional domains, the best studied of which is the C-terminal domain, containing several lysine-proline-alanine-rich repeats. This domain has been shown to be involved in mycobacterial binding to epithelial cells $[2,20]$. The BACTH studies described here show that it is not involved in binding to Rv0613c, since BACTH experiments done with a truncated version of HBHA, lacking this C-terminal domain, gave the same results as those obtained with full-length HBHA (Figure 1A,B). This implies that the HBHA-Rv0613c interaction is not based merely on ionic interactions of the positively charged C-terminal domain of HBHA with Rv0613c, which could lack specificity. It rather suggests a more specific protein-protein interaction. As HBHA is an intrinsically disordered protein [15], it may therefore be possible that its interaction with Rv0613c induces a specific conformation of HBHA enabling it to be properly exposed and oriented at the bacterial surface and to carry out its biological function as an adhesin and a bacterial clumping factor.

Both Rv0613c and MSMEG_1285 contain a SEC-C motif (Figure 2B), notably found in bacterial preprotein translocases. It is therefore tempting to speculate that Rv0613c and MSMEG_1285 fulfill a role similar to that of the preprotein translocase SecA and the chaperone SecB in order to facilitate HBHA 
export through the mycobacterial cytoplasmic membrane and HBHA cell surface exposure. Accordingly, the deletion of MSMEG_1285 led to the impairment of the proper cell surface exposure of recombinant HBHA_Mtb produced in Msmeg, as evidenced by AFM (Figure 3, Supplementary Figure S3). The deletion also led to a decrease in mycobacterial auto-aggregation by the HBHA_Mtb-producing Msmeg strain (Figure 4A), to a smooth colony phenotype when grown on Middlebrook 7H11 agar plates (Figure 4B) and to a decrease in infectivity of A549 cells (Figure 5A). Native HBHA has also been reported to form homodimers, and it was previously suggested that the HBHA trans-dimerization is responsible for bacterial agglutination [21]. Therefore, a defect in HBHA cell surface exposure and orientation would directly lead to the loss of the auto-aggregation, to a smooth phenotype, and a decrease in infectivity. Importantly, the formation of large mycobacterial aggregates has been reported to increase cytotoxicicty and to enhance effective killing of host cells after macrophage internalization, highlighting the importance of aggregation state in $M t b$ virulence [22].

Although Rv0613c has no significant predicted structural similarities with hydrolases or peptidases, it was recently shown to display protease activity, as evidenced by its ability to cleave $\beta$-casein [16]. It was suggested that either Rv0613c is a divergent protease or that its protease activity is a moonlighting function [16]. However, we have no evidence that Rv0613c exerts a protease activity on HBHA. Interestingly, the proteases tested by Ortega et al. [16], Rv0525 and Rv1192, with a molecular weight of 22 and $30 \mathrm{kDa}$, respectively, are three- to four-fold smaller than the 93-kDa Rv0613c, suggesting that Rv0613c may harbor several subdomains with different activities. In the BACTH assay, the portion of Rv0613c (Rv0613 $c_{314-550}$ ) interacting with HBHA represents only one quarter of the entire Rv0613c protein. Thus, the protease subdomain of Rv0613c could be distinct from the HBHA-interacting subdomain. It remains to be determined whether the protease activity of Rv0613c plays any role in HBHA maturation process.

In addition to the SEC-C motif, MSMEG_1285 also contains a TPR domain. In spite of some divergence in the N-terminal part, the C-terminal part of the MSMEG_1285 TPR domain is very similar to the corresponding sequence of Rv0613c (Figure 2B), strongly suggesting that Rv0613c also contains a TPR domain, although it was originally not annotated as such in protein databases. However, the web-based TPRpred tool (Available online: https://toolkit.tuebingen.mpg.de/\#/tools/tprpred) predicts that ${ }_{364}$ VAVRW ... MDTEW 397 and ${ }_{398}$ PLPLL ... EPDHP ${ }_{431}$ of Rv0613c are TPR peptides with a $p$-value of $1.6 \times 10^{-5}$ and $4.1 \times 10^{-4}$, respectively. This portion of Rv0613c is also present in the fragment identified by the BACTH system to interact with HBHA. TPR domains are known to mediate protein-protein interactions and TPR-containing proteins are often involved in the biogenesis of bacterial secreted or surface proteins [17]. For example, the assembly machinery of the $\beta$-barrel outer membrane protein of E. coli consists of a complex of five proteins, one of which, named BamD, contains 5 TPR domains [23]. This protein binds to unfolded outer membrane proteins prior to folding and insertion into the outer membrane [24,25]. ClustalO predicts $21 \%$ of sequence identity between the TPR1 of BamD and Rv0613c471-546 (Supplementary Figure S5), which is contained within the portion of Rv0613c that interacts with HBHA in the BACTH system. Similarly, the TPR-containing protein TprA from Porphyromonas gingivalis is also involved in the outer-membrane display of the TapA protein, most likely via protein-protein interaction involving the TPR domain of TprA and this mechanism is required to maintain full infectivity of epithelial cells by $P$. gingivalis [26]. Thus, it is tempting to speculate that a similar mechanism, involving protein-protein interactions with the TPR domain of Rv0613c, may be at play to address HBHA to the mycobacterial cell surface in its proper orientation. Interestingly, both SEC-C motif- and TPR domain-containing proteins are enriched in the pathogenic $M t b$ clade in comparison with non-pathogenic mycobacteria [27], which suggests their involvement in virulence traits.

It was of interest to investigate whether the sub-cellular localization of proteins was also affected by the deletion of MSMEG_1285. Surprisingly, an overrepresentation (cut-off $\geq 5$ ) of a single Msmeg protein, MSMEG_3496, was seen in the MB fraction of the mutant as compared to Msmeg $\mathrm{mc}^{2} 155$. This protein is one of the orthologues of MmpL5 (Rv0676c) in Mtb. It is intriguing that the second 
protein, MmpL14, found here to interact with HBHA in the BACTH system is also a member of the MmpL family. The mmpL14 gene is part of the RvD2 deleted region and is absent in Mtb H37Rv [12,13], but conserved among the majority of Mtb strains, including CDC1551 (locus MT1802) and H37Ra [28,29], as well as most of the sequenced $M t b$ clinical isolates. The MmpL protein family has been shown to be involved in substrate transport, such as the transport of lipids through the mycobacterial membrane, siderophore export and iron acquisition, and drug efflux [30,31]. MmpL14 ${ }_{519-749}$ comprises the complete docking subdomain DC as well as part of the porter subdomains PC1 and PC2, making this part of the protein accessible for interactions with surface-exposed proteins. The periplasmic docking subdomain DC was suggested to play a role in protein-protein interactions [32], consistent with our findings on its interaction with HBHA. Moreover, the rough to smooth transition phenotype cannot be reduced only to the impairment of HbhA transport. Interestingly, Bernut and coworkers reported that a $\mathrm{Y} 842 \mathrm{H}$ mutation in M. bolletii MmpL4a was involved in the smooth to rough transition phenotype [33]. The authors proposed that this single key mutation has an impact of the 3D structure of the protein, affecting the proton motive force. Considering the interaction of HBHA with MmpL14, the rough to smooth transition phenotype could be an indirect consequence of the improper HBHA cell surface exposure, also compromising the structural organization and/or exposure of MmpL proteins. In addition, as MmpL proteins are key players in the transport of lipids, the authors suggested that a functional link may exist between MmpL4a and the GPL biosynthesis [33]. We can therefore not exclude link between HBHA and the MmpL proteins during the biogenesis or function of HBHA. Future work will address this issue.

\section{Materials and Methods}

\subsection{Bacterial Strains and Media}

E. coli TOP10 (Invitrogen, Carlsbad, CA, USA) was used for cloning and E. coli DHM1 was used for BACTH assays (Table S1). The E. coli strains were grown in LB medium (MP Biomedicals, Illkirch, France) at $37^{\circ} \mathrm{C}$ supplemented with ampicillin $\left(100 \mu \mathrm{g} \cdot \mathrm{mL}^{-1}\right)$, hygromycin $\left(50 \mu \mathrm{g} \cdot \mathrm{mL}^{-1}\right)$ or kanamycin $\left(25 \mu \mathrm{g} \cdot \mathrm{mL}^{-1}\right)$, when required. Msmeg $\mathrm{mc}^{2} 155$, the deletion mutant $\triangle M S M E G \_1285$ and the corresponding complemented strain (Table S1) were grown in Sauton's medium (containing 0.025\% tyloxapol), in Middlebrook 7H9 broth or on 7H11 agar plates supplemented with OADC enrichment (Becton Dickinson, Le Pont-de-Claix, France), hygromycin $\left(50 \mu \mathrm{g} \cdot \mathrm{mL}^{-1}\right)$ or kanamycin $\left(25 \mu \mathrm{g} \cdot \mathrm{mL}^{-1}\right)$, when required.

\subsection{Generation of the $\triangle M S M E G \_1285$ Mutant in Msmeg $m c^{2} 155$}

To generate the $\triangle M S M E G \_1285$ mutant, we followed the protocol previously described by van Kessel et al. [34]. Briefly, upstream and downstream regions of $M S M E G \_1285$ were amplified from Msmeg $\mathrm{mc}^{2} 155$ genomic DNA using primers [Up_MSMEG1285_dir and Up_MSMEG1285_rev] and [Down_MSMEG1285_dir and Down_MSMEG1285_rev], respectively (Table S2). These upstream and downstream fragments were inserted at either side of a hygromycin-resistance cassette in pJSC347 (Table S1). After digestion by SpeI and StuI, the allelic exchange substrate was electroporated into competent Msmeg $\mathrm{mc}^{2} 155$ containing pJV53 (Table S1), following induction by $0.2 \%$ acetamide for $24 \mathrm{~h}$. Several hygromycin-resistant clones were selected and tested for the insertion of the hygromycin-resistance cassette by PCR using primers Seq_MSMEG1285_1, Seq_MSMEG1285_2 and Seq_MSMEG1285_3 (Supplementary Figure S2A,B and Table S2). The resulting PCR fragments were further checked by sequencing to confirm the insertion at the expected $M s m e g \mathrm{mc}^{2} 155$ locus.

\subsection{Plasmid Constructions}

Different portions of $h b h A$ were amplified by PCR using $M t b H 37 R v$ genomic DNA and the pair of primers [pKT25_hbhA_dir and pKT25_hbhA_rev] or [pKT25_hbhA_dir and pKT25_hbhA1-109_rev] (Table S2). The PCR fragments were digested with BamHI and KpnI and ligated into pKT25 
digested with the same enzymes, thereby generating pKT25_hbhA and pKT25_hbh $A_{1-109}$ (Table S1). The MSMEG_1285 gene was amplified by PCR using Msmeg $\mathrm{mc}^{2} 155$ genomic DNA and the pair of primers [pVV16_MSMEG1285_dir and pVV16_MSMEG1285_rev] (Table S2). The PCR fragment was digested with NdeI and HindIII and ligated into pVV16 digested with the same enzymes, thereby generating pVV16_MSMEG_1285 (Table S1). All plasmids were checked by sequencing.

\subsection{Bacterial Adenylate Cyclase Two-Hybrid Assays}

One hundred ng of the Mtb Erdman library contained in pUT18C was electroporated into E. coli DHM1 containing pKT25_hbhA. Transformants were spread onto MacConkey agar (Becton Dickinson) plates supplemented with $100 \mu \mathrm{g} \cdot \mathrm{mL}^{-1}$ ampicillin, $25 \mu \mathrm{g} \cdot \mathrm{mL}^{-1}$ kanamycin, $0.5 \mathrm{mM} \mathrm{IPTG}$ and $1 \%$ maltose. After 4 to 6 days at $30^{\circ} \mathrm{C}$, clones were selected based on their red color and transferred onto fresh MacConkey agar plates to confirm the red phenotype. DNA was extracted from the selected colonies and pUT18C inserts were sequenced using the Seq_pUT18C_dir primer (Table S2). To confirm the phenotype, pUT18C containing rv0613 $c_{314-550}$ was re-introduced into E. coli DHM1 containing either pKT25_hbhA or pKT25_hbhA 1 1-109. Transformants were spread onto both MacConkey agar plates and LB agar plates supplemented with $100 \mu \mathrm{g} \cdot \mathrm{mL}^{-1}$ ampicillin, $25 \mu \mathrm{g} \cdot \mathrm{mL}^{-1}$ kanamycin, $0.5 \mathrm{mM}$ IPTG and $50 \mu \mathrm{g} \cdot \mathrm{mL}^{-1} \mathrm{X}$-gal and incubated for 3 days at $30^{\circ} \mathrm{C}$.

\subsection{Fractionation Protocol}

Msmeg strains were disrupted in a French pressure cell and the lysates were centrifuged at $3000 \times g$ for $10 \mathrm{~min}$ at $4{ }^{\circ} \mathrm{C}$ to eliminate cellular debris and non-lysed cells. The supernatants were pelleted at $27,000 \times \mathrm{g}$ for $30 \mathrm{~min}$ at $4{ }^{\circ} \mathrm{C}$ in order to collect cell-wall fractions (CW). The remaining supernatants were centrifuged at $100,000 \times g$ for $2 \mathrm{~h}$ at $4{ }^{\circ} \mathrm{C}$ to separate the membrane fractions (MB) from cytosolic fractions. Each fraction was resuspended in an appropriate volume of Laemmli buffer.

\subsection{Immunoblot Analysis}

Proteins were resolved by SDS-PAGE using 12\% acrylamide gels and then electro-transferred onto a nitrocellulose membrane. The membrane was saturated with Tris-Buffered Saline (pH 7.5, TBS) containing $0.05 \%$ Tween 80 and $5 \%$ milk and probed overnight at $4{ }^{\circ} \mathrm{C}$ with anti-HBHA monoclonal antibody VF2 or D2 diluted 1:100 in TBS-Tween-3\% milk or sera from Rv0613c-immunized mice diluted 1:100 in TBS-Tween-3\% milk or anti-Hsp65 monoclonal antibody (Abcam, Cambridge, UK) diluted 1:1000 in TBS-Tween-3\% milk. Finally, the membrane was incubated for $1 \mathrm{~h}$ at room temperature with goat anti-mouse horseradish peroxidase-conjugated secondary antibodies (Abcam), diluted 1:5000 in TBS-Tween-3\% milk. Detection was performed using the Amersham ECL Prime Western-Blotting Detection Reagent (Fisher Scientific, Illkirch, France) and chemiluminescence was detected using the Amersham Imager 600 (GE Healthcare, Uppsala, Sweden).

\subsection{Atomic Force Microscopy}

Gold-coated cantilevers were cleaned with plasma oxygen, rinsed with ethanol, dried with a gentle nitrogen flow and immersed overnight at room temperature in a $25 \mu \mathrm{g} \cdot \mathrm{mL}^{-1}$ solution of biotinylated bovine serum albumin (BBSA) (Sigma, Saint-Quentin-Fallavier, France) in PBS. Following rinsing with PBS, the BBSA surfaces were exposed to a $10 \mu \mathrm{g} \cdot \mathrm{mL}^{-1}$ solution of streptavidin (Sigma) in PBS for $2 \mathrm{~h}$ followed by thorough rinsing with PBS. Finally, the BBSA/streptavidin surfaces were immersed for $2 \mathrm{~h}$ in PBS containing $10 \mu \mathrm{g} \cdot \mathrm{mL}^{-1}$ biotinylated heparin (Sigma) and rinsed with PBS. The mycobacteria were immobilized on porous polycarbonate membranes with a $3 \mu \mathrm{m}$ pore size (Merck Millipore, Darmstadt, Germany). After filtering a concentrated bacterial suspension, the membrane was transferred to a Petri dish containing the imaging solution and gently agitated to remove non-immobilized bacteria. Then, a small piece of the membrane $(1 \mathrm{~cm} \times 1 \mathrm{~cm}$ square $)$ was cut and the bottom side was quickly dried using precision wipes before being attached to a steel sample puck using a small piece of double side adhesive tape. The mounted samples were transferred into the 
AFM liquid cell while avoiding dewetting. AFM contact mode images and force-distance curves were obtained using a MultiMode 8 AFM (Bruker, Santa Barbara, CA, USA). Measurements were performed in PBS at room temperature using gold-coated Silicon Nitride cantilevers (OMCL-TR400PB, Olympus Ltd., Tokyo, Japan). The spring constants of the cantilevers were measured using the thermal noise method (NanoScope Analysis software version 8.15, Bruker), yielding values ranging from 0.022 to $0.026 \mathrm{~N} \cdot \mathrm{m}^{-1}$. All force measurements were performed using a constant approach and retraction speed of $1500 \mathrm{~nm} \cdot \mathrm{s}^{-1}$, and with an interaction time of $500 \mathrm{~ms}$. Adhesion maps were obtained by recording $16 \times 16$ force curves on $400 \mathrm{~nm} \times 400 \mathrm{~nm}$ areas of the cells, calculating the adhesion force values and displaying them as grey pixels. Histograms were obtained by pooling the data from several adhesion maps. Data analysis was done using in-house developed pyAF (python Atomic Force) software, version 1.5.1. All experiments were performed at least on four different bacteria and with two different heparin-coated AFM tips.

\subsection{Infection of A549 Cells}

One day prior to infection, $2 \times 10^{5}$ A549 cells per well resuspended in DMEM containing $10 \%$ Fetal Calf Serum (FCS) were seeded in 24-well plates and incubated overnight at $37{ }^{\circ} \mathrm{C}$ under $5 \% \mathrm{CO}_{2}$. Cultures of Msmeg $\mathrm{mc}^{2} 155, \triangle M S M E G \_1285$ and their recombinant HBHA_Mtb-producing derivatives were grown overnight at $37^{\circ} \mathrm{C}$ with shaking. Bacteria were washed twice in PBS, resuspended in DMEM containing $10 \%$ FCS, treated for $15 \mathrm{~min}$ in a sonication bath and centrifuged for $2 \mathrm{~min}$ at $120 \times g$ to eliminate remaining clumps. After two washes with PBS, A549 cells were infected with bacterial suspensions at an MOI of 50 and further incubated for $4 \mathrm{~h}$ at $37^{\circ} \mathrm{C}$ under $5 \% \mathrm{CO}_{2}$. After $4 \mathrm{~h}$, the cells were extensively washed 5 times with PBS and either resuspended in DMEM containing $10 \%$ FCS or lysed with $250 \mu \mathrm{L}$ of $0.1 \%$ Triton X-100. Serial dilutions of the lysates were plated onto 7H11 agar plates and $\mathrm{CFU}$ were counted after 3 days of incubation at $37^{\circ} \mathrm{C}$. At each time point, the remaining cells were washed 5 times in PBS and resuspended in fresh DMEM containing 10\% FCS or, alternatively, lysed and CFU counts determined similarly. Each measure was done in triplicate and each experiment was done independently at least twice.

\subsection{Mass Spectrometry Proteomic Analysis}

After heating at $100{ }^{\circ} \mathrm{C}$ in 5\% SDS, 5\% $\beta$-mercaptoethanol, $1 \mathrm{mM}$ EDTA, $10 \%$ glycerol, $10 \mathrm{mM}$ Tris- $\mathrm{HCl}(\mathrm{pH} 8)$ for $3 \mathrm{~min}$, protein samples were fractionated by SDS-PAGE on a $10 \%$ acrylamide gel. The electrophoretic migration was stopped as soon as the protein sample entered $0.3 \mathrm{~cm}$ into the separating gel. The gel was briefly stained with Coomassie Blue, and one band, containing the entire sample, was cut. In-gel digestion of gel slices was performed as previously described [35]. The UltiMate 3000 RSLCnano System (Fisher Scientific) was used for the separation of the protein digests. Peptides were automatically fractionated onto a commercial C18 reversed phase column $\left(75 \mu \mathrm{m} \times 250 \mathrm{~mm}, 2 \mu \mathrm{m}\right.$ particle, PepMap100 RSLC column, Thermo Fisher Scientific, at $\left.35^{\circ} \mathrm{C}\right)$. Trapping was performed during $4 \mathrm{~min}$ at $5 \mu \mathrm{L} \cdot \mathrm{min}^{-1}$, with solvent $\mathrm{A}\left(98 \% \mathrm{H}_{2} \mathrm{O}, 2 \%\right.$ acetonitrile and $0.1 \%$ formic acid). The peptides were eluted using two solvents $\mathrm{A}(0.1 \%$ formic acid in water $)$ and $\mathrm{B}\left(0.1 \%\right.$ formic acid in acetonitrile) at a flow rate of $300 \mathrm{~nL} \cdot \mathrm{min}^{-1}$. Gradient separation was $3 \mathrm{~min}$ at $3 \% \mathrm{~B}, 110 \mathrm{~min}$ from $3 \% \mathrm{~B}$ to $20 \% \mathrm{~B}, 10 \mathrm{~min}$ from $20 \% \mathrm{~B}$ to $80 \% \mathrm{~B}$ and maintained for $15 \mathrm{~min}$ at $80 \%$ B. The column was equilibrated for 6 min with $3 \%$ buffer B prior to the next sample analysis. Eluted peptides from the C18 column were analyzed by Q-Exactive instruments (Fisher Scientific). The electrospray voltage was $1.9 \mathrm{kV}$, and the capillary temperature was $275^{\circ} \mathrm{C}$. Full MS scans were acquired in the Orbitrap mass analyzer over $m / z$ 400-1200 range with a 70,000 ( $/ \mathrm{m} / z 200)$ resolution. The target value was $3 \times 10^{6}$. Fifteen most intense peaks with charge state between 2 and 5 were fragmented in the higher-energy collision-activated dissociation cell with normalized collision energy of $27 \%$, and tandem mass spectrum was acquired in the Orbitrap mass analyzer with a $17,500(\mathrm{~m} / \mathrm{z} 200)$ resolution. The target value was $1 \times 10^{5}$. The ion selection threshold was $5 \times 10^{4}$ counts, and the 
maximum allowed ion accumulation times were $250 \mathrm{~ms}$ for full MS scans and $100 \mathrm{~ms}$ for tandem mass spectrum. Dynamic exclusion was set to $30 \mathrm{~s}$.

\subsection{Proteomic Data Analysis}

Raw data collected during nanoLC-MS/MS analyses were processed and converted into a *.mgf peak list format with Proteome Discoverer 1.4 (Fisher Scientific). MS/MS data were analyzed using search engine Mascot (version 2.4.0, Matrix Science, London, UK) installed on a local server. Searches were performed with a tolerance on mass measurement of $0.2 \mathrm{Da}$ for precursor and $0.2 \mathrm{Da}$ for fragment ions, against a composite target-decoy database (17,848 total entries) built with a Msmeg UniProt database (strain ATCC 700084/mc (2)155, taxo 246196, October 2014, 8805 entries) fused with the sequences of HBHA_EGFP, recombinant trypsin and a list of classical contaminants (119 entries). Cysteine carbamidomethylation, methionine oxidation, protein N-terminal acetylation and cysteine propionamidation were searched as variable modifications. Up to one missed trypsin cleavage was allowed. For each sample, peptides were filtered out according to the cutoff set for protein hits with 1 or more peptides larger than 8 residues, ion score $>25$, identity score $>6$, corresponding to a $1 \%$ false positive rate.

\subsection{Statistical Analysis}

For infection of A549 cells by the Msmeg strains, the statistical significance ( $p$-value) was tested with a two-tailed unpaired $t$-test (GraphPad Prism version 5.04, GraphPad Software, CA, USA) and $p$-values $<0.05$ were considered as significant.

Supplementary Materials: Supplementary materials can be found at http:/ /www.mdpi.com/1422-0067/19/6/ 1673/s1.

Author Contributions: R.V.-C. and D.R. conceived and designed the study. R.V-C., V.D., J.-M.S. and F.L. acquired, analyzed and interpreted the data. R.V.-C. and C.L. wrote the manuscript.

Acknowledgments: We thank Alain Baulard (Institut Pasteur de Lille, France) for kindly providing the Mtb Erdman library in pUT18C vector and Carine Rouanet (Institut Pasteur de Lille, France) for providing the sera from Rv0613c-immunized mice. We acknowledge the BioImaging Center Lille (BICeL) for access to equipment. This work has been supported by ANR 10-EQPX-04-01 and FEDER 12,001,407 to BICeL. This work was supported in part by the European Commission Horizon 2020 (TBVAC2020, H2020-PHC-643381).

Conflicts of Interest: The authors declare no conflict of interest.

\section{References}

1. WHO. World Health Organization Global Tuberculosis Report; WHO: Geneva, Switzerland, 2017.

2. Menozzi, F.D.; Rouse, J.H.; Alavi, M.; Laude-Sharp, M.; Muller, J.; Bischoff, R.; Brennan, M.J.; Locht, C. Identification of a heparin-binding hemagglutinin present in mycobacteria. J. Exp. Med. 1996, 184, 993-1001. [CrossRef] [PubMed]

3. Menozzi, F.D.; Bischoff, R.; Fort, E.; Brennan, M.J.; Locht, C. Molecular characterization of the mycobacterial heparin-binding hemagglutinin, a mycobacterial adhesin. Proc. Natl. Acad. Sci. USA 1998, 95, 12625-12630. [CrossRef] [PubMed]

4. Huang, T.Y.; Irene, D.; Zulueta, M.M.; Tai, T.J.; Lain, S.H.; Cheng, C.P.; Tsai, P.X.; Lin, S.Y.; Chen, Z.G.; $\mathrm{Ku}, \mathrm{C} . \mathrm{C}$; et al. Structure of the Complex between a Heparan Sulfate Octasaccharide and Mycobacterial Heparin-Binding Hemagglutinin. Angew. Chem. Int. Ed. Engl. 2017, 56, 4192-4196. [CrossRef] [PubMed]

5. Pethe, K.; Alonso, S.; Biet, F.; Delogu, G.; Brennan, M.J.; Locht, C.; Menozzi, F.D. The heparin-binding haemagglutinin of $M$. tuberculosis is required for extrapulmonary dissemination. Nature 2001, 412, 190-194. [CrossRef] [PubMed]

6. Zimmermann, N.; Saiga, H.; Houthuys, E.; Moura-Alves, P.; Koehler, A.; Bandermann, S.; Dorhoi, A.; Kaufmann, S.H. Syndecans promote mycobacterial internalization by lung epithelial cells. Cell. Microbiol. 2016, 18, 1846-1856. [CrossRef] [PubMed] 
7. Pethe, K.; Bifani, P.; Drobecq, H.; Sergheraert, C.; Debrie, A.S.; Locht, C.; Menozzi, F.D. Mycobacterial heparin-binding hemagglutinin and laminin-binding protein share antigenic methyllysines that confer resistance to proteolysis. Proc. Natl. Acad. Sci. USA 2002, 99, 10759-10764. [CrossRef] [PubMed]

8. Temmerman, S.; Pethe, K.; Parra, M.; Alonso, S.; Rouanet, C.; Pickett, T.; Drowart, A.; Debrie, A.S.; Delogu, G.; Menozzi, F.D.; et al. Methylation-dependent T cell immunity to Mycobacterium tuberculosis heparin-binding hemagglutinin. Nat. Med. 2004, 10, 935-941. [CrossRef] [PubMed]

9. Biet, F.; Angela de Melo Marques, M.; Grayon, M.; Xavier da Silveira, E.K.; Brennan, P.J.; Drobecq, H.; Raze, D.; Vidal Pessolani, M.C.; Locht, C.; Menozzi, F.D. Mycobacterium smegmatis produces an HBHA homologue which is not involved in epithelial adherence. Microbes Infect. 2007, 9, 175-182. [CrossRef] [PubMed]

10. Lanfranconi, M.P.; Alvarez, H.M. Functional divergence of HBHA from Mycobacterium tuberculosis and its evolutionary relationship with TadA from Rhodococcus opacus. Biochimie 2016, 127, 241-248. [CrossRef] [PubMed]

11. Karimova, G.; Pidoux, J.; Ullmann, A.; Ladant, D. A bacterial two-hybrid system based on a reconstituted signal transduction pathway. Proc. Natl. Acad. Sci. USA 1998, 95, 5752-5756. [CrossRef] [PubMed]

12. Gordon, S.V.; Brosch, R.; Billault, A.; Garnier, T.; Eiglmeier, K.; Cole, S.T. Identification of variable regions in the genomes of tubercle bacilli using bacterial artificial chromosome arrays. Mol. Microbiol. 1999, 32, 643-655. [CrossRef] [PubMed]

13. Domenech, P.; Reed, M.B.; Barry, C.E., 3rd. Contribution of the Mycobacterium tuberculosis MmpL protein family to virulence and drug resistance. Infect. Immun. 2005, 73, 3492-3501. [CrossRef] [PubMed]

14. Pethe, K.; Aumercier, M.; Fort, E.; Gatot, C.; Locht, C.; Menozzi, F.D. Characterization of the heparin-binding site of the mycobacterial heparin-binding hemagglutinin adhesin. J. Biol. Chem. 2000, 275, 14273-14280. [CrossRef] [PubMed]

15. Lebrun, P.; Raze, D.; Fritzinger, B.; Wieruszeski, J.M.; Biet, F.; Dose, A.; Carpentier, M.; Schwarzer, D.; Allain, F.; Lippens, G.; et al. Differential contribution of the repeats to heparin binding of HBHA, a major adhesin of Mycobacterium tuberculosis. PLoS ONE 2012, 7, e32421. [CrossRef] [PubMed]

16. Ortega, C.; Anderson, L.N.; Frando, A.; Sadler, N.C.; Brown, R.W.; Smith, R.D.; Wright, A.T.; Grundner, C. Systematic Survey of Serine Hydrolase Activity in Mycobacterium tuberculosis Defines Changes Associated with Persistence. Cell. Chem. Biol. 2016, 23, 290-298. [CrossRef] [PubMed]

17. Cerveny, L.; Straskova, A.; Dankova, V.; Hartlova, A.; Ceckova, M.; Staud, F.; Stulik, J. Tetratricopeptide repeat motifs in the world of bacterial pathogens: Role in virulence mechanisms. Infect. Immun. 2013, 81, 629-635. [CrossRef] [PubMed]

18. Dupres, V.; Menozzi, F.D.; Locht, C.; Clare, B.H.; Abbott, N.L.; Cuenot, S.; Bompard, C.; Raze, D.; Dufrene, Y.F. Nanoscale mapping and functional analysis of individual adhesins on living bacteria. Nat. Methods 2005, 2, 515-520. [CrossRef] [PubMed]

19. Raze, D.; Verwaerde, C.; Deloison, G.; Werkmeister, E.; Coupin, B.; Loyens, M.; Brodin, P.; Rouanet, C.; Locht, C. Heparin-binding Hemagglutinin Adhesin (HBHA) is involved in intracytosolic lipid inclusions (ILI) formation in mycobacteria. Manuscript in preparation, 2018.

20. Delogu, G.; Brennan, M.J. Functional domains present in the mycobacterial hemagglutinin, HBHA. J. Bacteriol. 1999, 181, 7464-7469. [PubMed]

21. Esposito, C.; Carullo, P.; Pedone, E.; Graziano, G.; Del Vecchio, P.; Berisio, R. Dimerisation and structural integrity of Heparin Binding Hemagglutinin A from Mycobacterium tuberculosis: Implications for bacterial agglutination. FEBS Lett. 2010, 584, 1091-1096. [CrossRef] [PubMed]

22. Mahamed, D.; Boulle, M.; Ganga, Y.; Mc Arthur, C.; Skroch, S.; Oom, L.; Catinas, O.; Pillay, K.; Naicker, M.; Rampersad, S.; et al. Intracellular growth of Mycobacterium tuberculosis after macrophage cell death leads to serial killing of host cells. Elife 2017, 6, e22028. [PubMed]

23. Noinaj, N.; Gumbart, J.C.; Buchanan, S.K. The beta-barrel assembly machinery in motion. Nat. Rev. Microbiol. 2017, 15, 197-204. [CrossRef] [PubMed]

24. Albrecht, R.; Zeth, K. Structural basis of outer membrane protein biogenesis in bacteria. J. Biol. Chem. 2011, 286, 27792-27803. [CrossRef] [PubMed]

25. Sandoval, C.M.; Baker, S.L.; Jansen, K.; Metzner, S.I.; Sousa, M.C. Crystal structure of BamD: An essential component of the beta-Barrel assembly machinery of gram-negative bacteria. J. Mol. Biol. 2011, 409, 348-357. [CrossRef] [PubMed] 
26. Kondo, Y.; Ohara, N.; Sato, K.; Yoshimura, M.; Yukitake, H.; Naito, M.; Fujiwara, T.; Nakayama, K. Tetratricopeptide repeat protein-associated proteins contribute to the virulence of Porphyromonas gingivalis. Infect. Immun. 2010, 78, 2846-2856. [CrossRef] [PubMed]

27. McGuire, A.M.; Weiner, B.; Park, S.T.; Wapinski, I.; Raman, S.; Dolganov, G.; Peterson, M.; Riley, R.; Zucker, J.; Abeel, T.; et al. Comparative analysis of Mycobacterium and related Actinomycetes yields insight into the evolution of Mycobacterium tuberculosis pathogenesis. BMC Genom. 2012, 13, 120. [CrossRef] [PubMed]

28. Fleischmann, R.D.; Alland, D.; Eisen, J.A.; Carpenter, L.; White, O.; Peterson, J.; DeBoy, R.; Dodson, R.; Gwinn, M.; Haft, D.; et al. Whole-genome comparison of Mycobacterium tuberculosis clinical and laboratory strains. J. Bacteriol. 2002, 184, 5479-5490. [CrossRef] [PubMed]

29. Zheng, H.; Lu, L.; Wang, B.; Pu, S.; Zhang, X.; Zhu, G.; Shi, W.; Zhang, L.; Wang, H.; Wang, S.; et al. Genetic basis of virulence attenuation revealed by comparative genomic analysis of Mycobacterium tuberculosis strain H37Ra versus H37Rv. PLoS ONE 2008, 3, e2375. [CrossRef] [PubMed]

30. Chalut, C. MmpL transporter-mediated export of cell-wall associated lipids and siderophores in mycobacteria. Tuberculosis 2016, 100, 32-45. [CrossRef] [PubMed]

31. Viljoen, A.; Dubois, V.; Girard-Misguich, F.; Blaise, M.; Herrmann, J.L.; Kremer, L. The diverse family of MmpL transporters in mycobacteria: From regulation to antimicrobial developments. Mol. Microbiol. 2017, 104, 889-904. [CrossRef] [PubMed]

32. Chim, N.; Torres, R.; Liu, Y.; Capri, J.; Batot, G.; Whitelegge, J.P.; Goulding, C.W. The Structure and Interactions of Periplasmic Domains of Crucial MmpL Membrane Proteins from Mycobacterium tuberculosis. Chem. Biol. 2015, 22, 1098-1107. [CrossRef] [PubMed]

33. Bernut, A.; Viljoen, A.; Dupont, C.; Sapriel, G.; Blaise, M.; Bouchier, C.; Brosch, R.; de Chastellier, C.; Herrmann, J.L.; Kremer, L. Insights into the smooth-to-rough transitioning in Mycobacterium bolletii unravels a functional Tyr residue conserved in all mycobacterial MmpL family members. Mol. Microbiol. 2016, 99, 866-883. [CrossRef] [PubMed]

34. Van Kessel, J.C.; Marinelli, L.J.; Hatfull, G.F. Recombineering mycobacteria and their phages. Nat. Rev. Microbiol. 2008, 6, 851-857. [CrossRef] [PubMed]

35. Miguet, L.; Bechade, G.; Fornecker, L.; Zink, E.; Felden, C.; Gervais, C.; Herbrecht, R.; Van Dorsselaer, A.; Mauvieux, L.; Sanglier-Cianferani, S. Proteomic analysis of malignant B-cell derived microparticles reveals CD148 as a potentially useful antigenic biomarker for mantle cell lymphoma diagnosis. J. Proteome Res. 2009, 8, 3346-3354. [CrossRef] [PubMed] 